\title{
Direct observation of Anderson localization in plasmonic terahertz devices
}

\begin{abstract}
Shashank Pandey ${ }^{1}$, Barun Gupta ${ }^{1}$, Sushil Mujumdar ${ }^{2}$ and Ajay Nahata ${ }^{1}$
We present the first experimental observation of Anderson localization in the terahertz frequency range using plasmonic structures. To accomplish this goal, we designed $\mathrm{THz}$ waveguides consisting of a one-dimensional array of rectangular apertures that were fabricated in a freestanding metal foil. Disorder is introduced into the waveguide by offsetting the position of each aperture by a random distance within a prescribed range. For example, for a waveguide with apertures spaced by $250 \mu \mathrm{m}$ in a periodic waveguide, $10 \%$ disorder would correspond to the apertures being shifted by a random value between $\pm 25 \mu \mathrm{m}$ along the waveguide axis. We find that for disorder levels below $25 \%$, there is only an increase in the propagation loss along the device. However, for two specific waveguides with $25 \%$ disorder, we observe a spatially localized mode that lies just within the stop band of the device and exhibits a double-sided exponential spatial decay away from the maximum.
\end{abstract}

Light: Science \& Applications (2017) 6, e16232; doi:10.1038/lsa.2016.232; published online 10 March 2017

Keywords: Anderson localization; disordered medium; 1D aperiodic lattice; terahertz plasmonics; terahertz waveguides; time domain spectroscopy

\section{INTRODUCTION}

The Anderson localization of waves is an exotic phenomenon in which a propagating wave is spatially localized purely by structural disorder, thereby inhibiting its transport across the system ${ }^{1-19}$. Although the generality of this wave phenomenon has been proven by its demonstration in acoustic waves ${ }^{5}$, matter waves ${ }^{6}$ and electromagnetic waves over different frequency ranges ${ }^{7-17}$, it has remained elusive in the terahertz domain. Here, we describe the direct observation of Anderson localization in the $\mathrm{THz}$ spectral range using $\mathrm{THz}$ plasmonic waveguides. These devices are built using periodically spaced structures fabricated into a metal foil, thus allowing surface-bound guidedwave modes below the Bragg frequency. We find that in the presence of appropriate positional disorder, a localized mode beyond the Bragg frequency becomes evident and exhibits a double-sided exponential spatial decay of the $\mathrm{THz}$ electric field. These results dramatically expand the spectral range, types of materials and types of applications in which localization may have an important role.

The experimental demonstration of the mesoscopic electromagnetic phenomenon of Anderson localization is known to be challenging, particularly in three-dimensional systems, wherein a critical degree of disorder is required to manifest localization ${ }^{17}$. In lower dimensions, the fact that all states are localized favors the experimental observation of disorder-induced localization, as long as an appropriate sample size is employed ${ }^{16}$. Even in low dimensions, a 'terahertz gap' remains in the literature on localization because dielectric materials, which are commonly employed in such demonstrations, are lossy in this spectral range $\mathrm{e}^{20,21}$, creating a situation whereby the absorption length may be shorter than the localization length. Metals, by contrast, are characterized by high conductivity and enable the low-loss propagation of surface plasmon polaritons $(\mathrm{SPPs})^{22-24}$. In this study, we employ metal foils that are periodically structured, which have been shown to support the low-loss propagation of narrowband $\mathrm{THz}$-guided-wave SPP modes ${ }^{25-27}$. In the simplest embodiment, we have shown that rectangular apertures that fully ${ }^{25}$ or partially ${ }^{27}$ perforate the metal foil can guide multiple narrowband sets of frequencies. Here, the in-plane length of the aperture orthogonal to the propagation direction determines the frequency of the lowest-order propagation mode, whereas the higher-order modes can be modeled as cavity modes of the aperture, assuming an effective depth ${ }^{25}$. The aperture spacing along the waveguide axis determines the Bragg frequency of the periodic structure, which creates an effective band-edge for the system. Only modes that are characterized by frequencies below the Bragg frequency are observed as propagating modes.

\section{MATERIALS AND METHODS}

In this investigation, we examine the guided-wave properties of $\mathrm{THz}$ waveguides in which disorder is introduced in the position of the apertures along the propagation direction. The propagating $\mathrm{THz}$ electric field is characterized using $\mathrm{THz}$ time-domain spectroscopy, which yields both amplitude and phase information. Broadband $\mathrm{THz}$

${ }^{1}$ Department of Electrical and Computer Engineering, University of Utah, Salt Lake City, UT 84112, USA and ${ }^{2}$ Tata Institute for Fundamental Research, Mumbai 400005, India Correspondence: A Nahata, Email: nahata@ece.utah.edu

Received 14 March 2016; revised 14 September 2016; accepted 26 September 2016; accepted article preview online 27 September 2016 
radiation extending from $\sim 0.1$ to $1.5 \mathrm{THz}$ is coupled to the waveguide using a single groove etched into the metal foil. The time-domain propagation properties of the coupled $\mathrm{THz}$ electric field can be measured at any point on or above the metal surface via electrooptic sampling by simply moving the detection crystal and the optical probe beam ${ }^{28}$. When $<110>\mathrm{ZnTe}$ is used as the detection medium, we only measure the $z$-component (normal to the metal surface) of the electric field, although other probe beam polarizations and crystal orientations can be used to measure the other electric field vector components $^{28,29}$.

In Figure 1a, we show a schematic of the plasmonic waveguide, in which the apertures are periodically spaced, along with the experimental excitation and measurement geometry. Disorder is introduced into these waveguides by randomly varying the positions of each aperture by a fractional amount, $\sigma$, along the propagation direction. For example, in the periodic waveguides discussed below, the aperture spacing was set to $250 \mu \mathrm{m}$. Thus, a waveguide with $\sigma=10 \%$ disorder means that each aperture would be shifted by a randomly determined distance between $\pm 25 \mu \mathrm{m}$ from its original position in the periodic array. A schematic diagram for a waveguide with $\sigma=25 \%$ is shown in Figure 1b. Because Anderson localization is a stochastic process, we designed eight different waveguide samples for each of nine levels of disorder, $\sigma$, varying between 0 and $25 \%$. All of the waveguides were fabricated via laser ablation in $750-\mu \mathrm{m}$-thick stainless steel foils. The measured position tolerance for the apertures was less than $5 \mu \mathrm{m}$. The $\mathrm{THz}$ time-domain signal was initially measured every $5 \mathrm{~mm}$ along each waveguide, with smaller measurement increments for the samples that showed evidence of localization. Detailed information about the fabrication of the waveguides and the measurement scheme is presented in the Supplementary Information.

\section{RESULTS AND DISCUSSION}

Figure 2 presents our measurement of $\mathrm{THz}$ wave propagation through the waveguides with varying degrees of disorder. In Figure 2a, the red profile shows the transmission spectrum measured at the $8 \mathrm{~cm}$ terminus from the waveguide input for the periodic sample. The spectrum illustrates three dominant modes, where the lowest-order mode is determined by the fundamental resonance of the subwavelength aperture, $\nu=c / 2 s=0.3 \mathrm{THz}$, and the next two higher-order modes are related to Fabry-Perot resonances within the apertures ${ }^{24}$. Transmission through the waveguide arises from coupling of the $\mathrm{THz}$ electric field between subwavelength apertures. The manifestation of other modes associated with higher-order resonances is forbidden by the band-edge. The corresponding Bragg frequency, in our case, is given by $c / 2 d=0.6 \mathrm{THz}$, beyond which no transmittance is seen to occur, despite the significant power beyond $0.6 \mathrm{THz}$ in the input pulse shown in Supplementary Information. Figure 2a also depicts configurationally averaged transmittance spectra for $\sigma=3 \%$ through $\sigma=25 \%$. In these data, the spectrum for $\sigma=25 \%$ is averaged over six devices, and the rest are averaged over all eight devices. These additional spectral measurements demonstrate that the introduction of disorder does not alter the spectral properties of the guided-wave modes for almost all of the waveguides and, in particular, does not necessarily remove the frequency constraints imposed by the Bragg frequency.

For planar (unperforated) stainless steel metal foils, we have previously found that the 1/e THz electric field decay length for SPPs along the surface of the metal is $21 \mathrm{~cm}$ at $0.3 \mathrm{THz}(12.6 \mathrm{~cm}$ at $0.5 \mathrm{THz}$ and $6.3 \mathrm{~cm}$ at $1 \mathrm{THz})^{24}$. Perforating the metal foil to create a waveguide based on periodically spaced apertures creates an additional loss mechanism. In Figure 2b, we show the magnitude of the $\mathrm{THz}$ electric field measured for waveguides with periodically spaced apertures $(\sigma=0 \%)$ at the peak of the lowest-order resonance $(0.3 \mathrm{THz})$. The corresponding 1/e propagation length is $8.2 \mathrm{~cm}$, which is still longer than the device length used in this study. Figure $2 \mathrm{~b}$ also shows the electric field attenuation for two other sets of waveguides with increased disorder. The corresponding 1/e decay lengths for each value of $\sigma$ are shown in Figure 2c, which appears to follow an approximately exponential dependence for reasons hitherto unknown to us. For each waveguide, we can model the amplitude at any
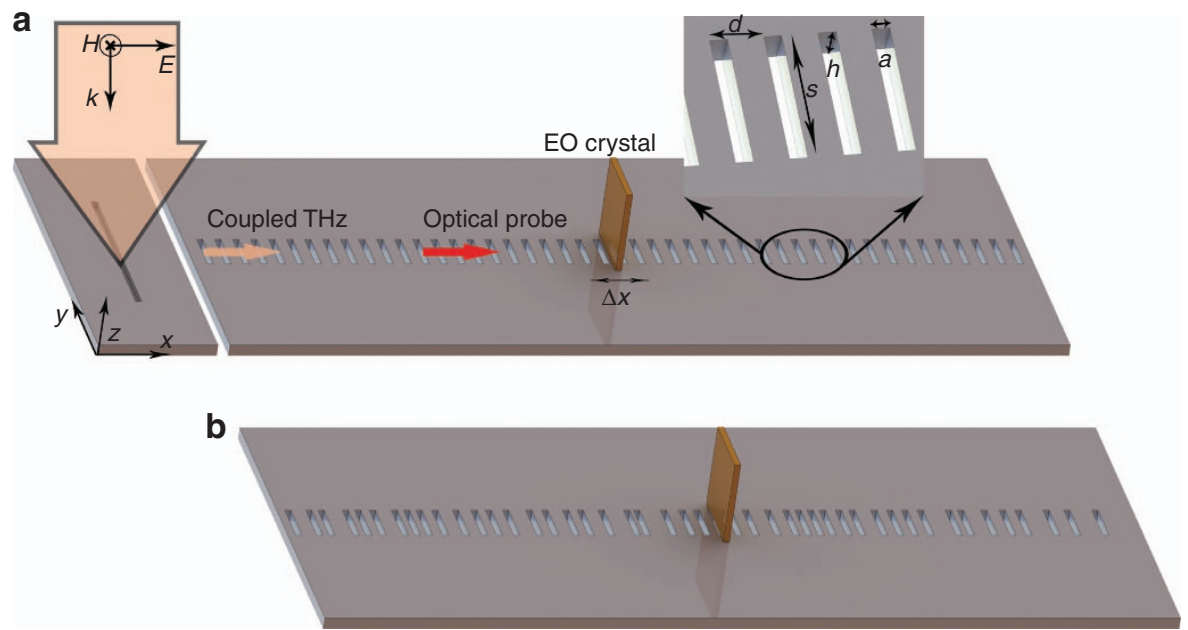

Figure 1 Schematic diagram of the THz waveguide, including the excitation and detection scheme. (a) Broadband THz radiation is normally incident on a 2$\mathrm{cm}$-long rectangular cross-section groove, $300 \mu \mathrm{m}$ wide by $100 \mu \mathrm{m}$ deep. The groove is used to couple the normally incident freely propagating broadband $\mathrm{THz}$ radiation into SPP waves that propagate along the sample surface. The 8 -cm-long waveguide consists of periodically spaced ( $\sigma=0 \%$ ) rectangular apertures with the following dimensions: $s=500 \mu \mathrm{m}, a=150 \mu \mathrm{m}, h=750 \mu \mathrm{m}$ and $d=250 \mu \mathrm{m}$. A $<110>Z \mathrm{nTe}$ crystal that can be freely positioned anywhere above the sample surface is used to measure the $z$-component of the THz electric field via electro-optic sampling. (b) Waveguide with identical aperture dimensions and $\sigma=25 \%$. 
a

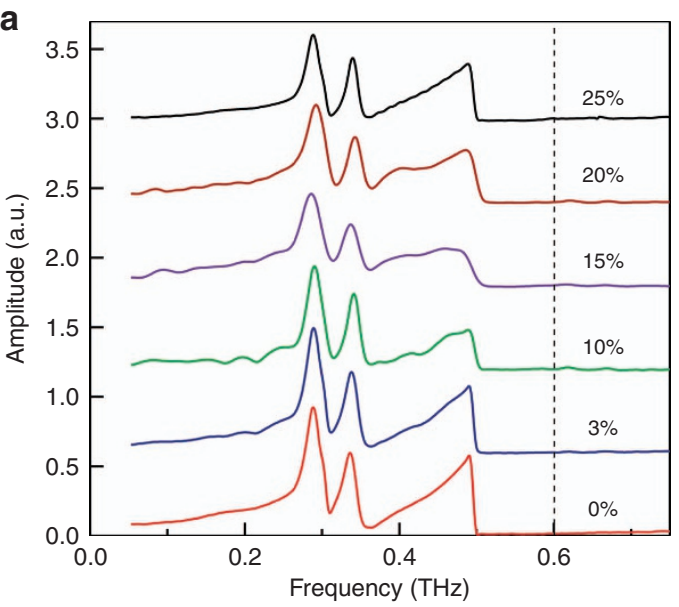

b

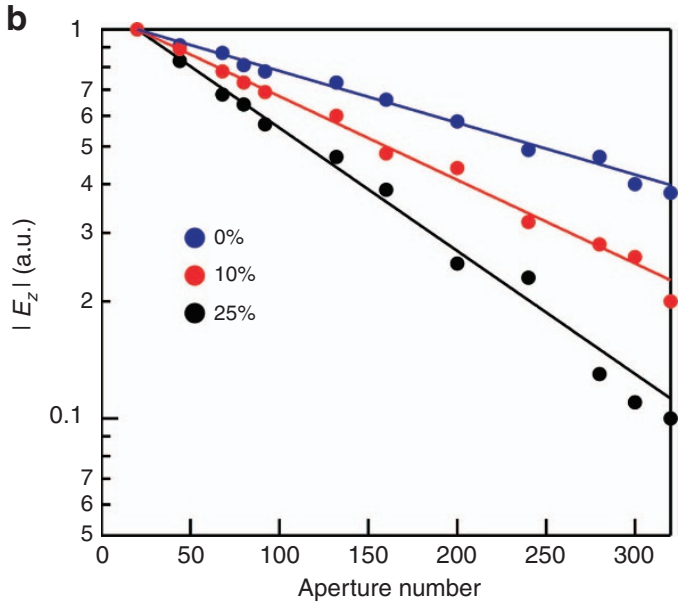

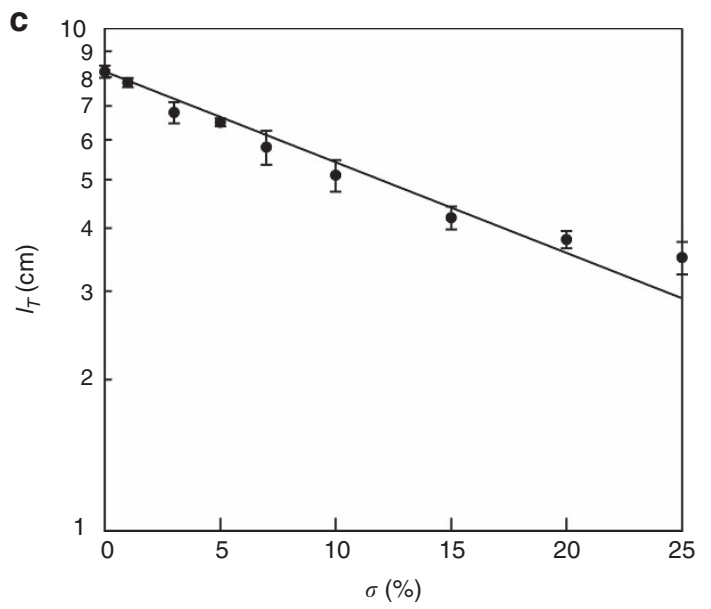

Figure 2 Propagation properties of the waveguides. (a) Experimentally measured transmission spectra for waveguides with disorder values ranging from $\sigma=0 \%$ to $\sigma=25 \%$ at a distance of $2 \mathrm{~cm}$ from the input groove. The spectra are the averages for all eight waveguides at each disorder level, except $\sigma=25 \%$, where the spectra have been averaged for only six of the eight devices. The dashed vertical line corresponds to the Bragg frequency at $0.6 \mathrm{THz}$. (b) Amplitude at the peak of the lowest-order resonance $(0.28 \mathrm{THz})$ along the length of the waveguide for $\sigma=0,10 \%$ and $25 \%$. The data are averaged as in a. The lines show the least squares fits to the data. (c) The total decay length $\ell_{T}$ for the lowest-order mode as a function of $\sigma$.
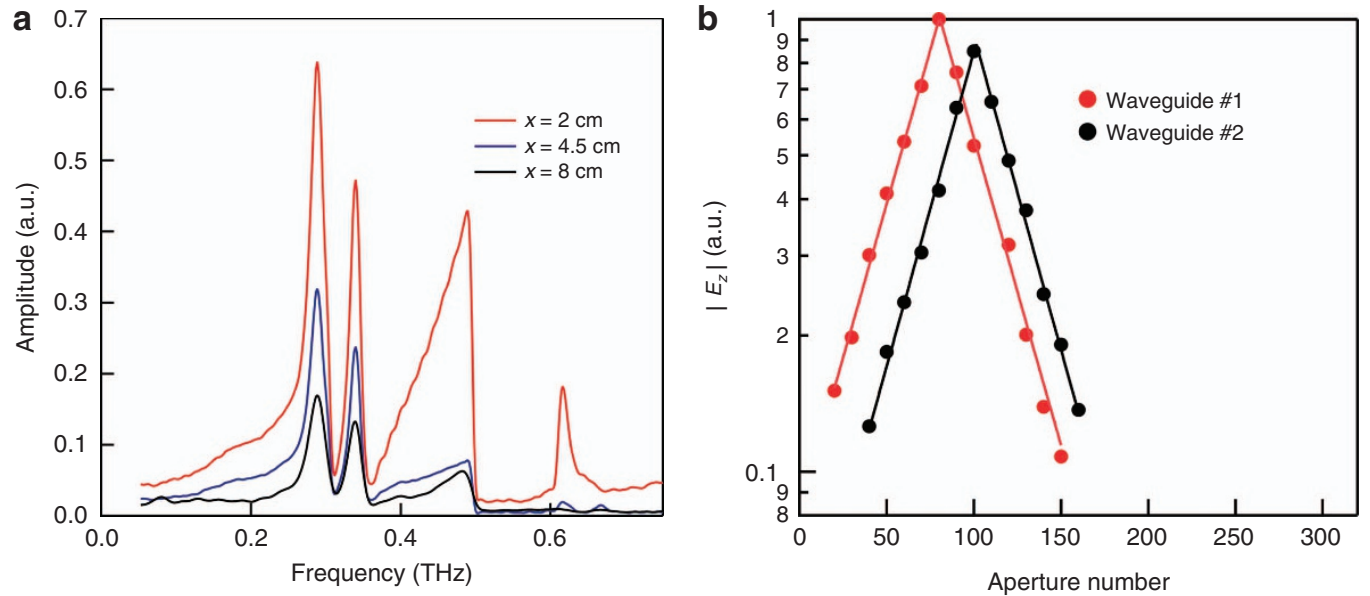

Figure 3 Spectral and spatial properties for two waveguides with $\sigma=25 \%$. (a) Transmission spectra of one of the two waveguides at distances of 2, 4.5 and $8 \mathrm{~cm}$ from the start of the waveguide. (b) Amplitude at the peak of the mode at $\sim 0.62 \mathrm{THz}$ for both waveguides (red and black dots) measured at $2.5 \mathrm{~mm}$ intervals about the maximum. The lines represent the least squares fits to the data for the two waveguides. 
given frequency using an exponential decay of the form $E_{z}(x)=E_{0} \exp \left[-x / \ell_{T}\right]$, where $x$ is the coordinate along the length of the waveguide and $\ell_{T}$ is the 1/e decay length. For $\sigma=25 \%, \ell_{T}$ for the lowest-order mode corresponds to $\sim 34 \lambda(3.5 \mathrm{~cm})$. Assuming that $\ell_{T}^{-1}=\ell_{\text {per }}^{-1}+\ell_{\text {dis }}^{-1}$, where $\ell_{\text {per }}$ is the decay length in a periodic waveguide $(8.2 \mathrm{~cm})$ and $\ell_{\text {dis }}$ is the decay length associated with the disorder, we obtain the disorder-induced decay length $\ell_{\text {dis }}=6.1 \mathrm{~cm}$, which is still comparable to the sample length for $\sigma=25 \%$. If we consider the next two higher-order modes for the same disordered waveguides $(\sigma=25 \%), \ell_{T}$ is $\sim 38 \lambda$ for both resonances at 0.34 and $0.49 \mathrm{THz}$.

As noted above, we only showed the spectra and spatial decay properties for six of the eight waveguides with $\sigma=25 \%$. The remaining two waveguides exhibited an additional resonance just beyond the Bragg frequency in the effective bandgap region. In Figure $3 \mathrm{a}$, we show the spectra for one of the two waveguides (because they exhibit very similar spectral properties) as a function of the spatial coordinate. For both of these waveguides, the spectral properties of the three propagating modes are nearly identical to the spectral properties of the other six waveguides with $\sigma=25 \%$, as are the spatial decay properties for the lowest-order mode. However, the decay properties for the additional mode at $\sim 0.62 \mathrm{THz}$ differ dramatically. In Figure 3b, we show the spatial properties of this new mode for both waveguides, which is clearly seen to peak in the middle of the samples away from the edges and is strongly localized over $\sim 100$ apertures. We fit the spatial decay properties of this mode in both waveguides at $0.62 \mathrm{THz}$ to a double-sided exponential decay of the form $E_{z}(x)=E_{\mathrm{o}} \exp \left[-\left|x-x_{\mathrm{o}}\right| / \xi\right]$, where $x_{\mathrm{o}}$ is the peak position of the mode and $\xi$ is the $1 / e$ decay length. In both cases, we find that $\xi$ is $\sim 8 \mathrm{~mm}(\sim 16 \lambda$ at $0.62 \mathrm{THz})$, which is much smaller than both $\ell_{T}$ and $\ell_{\text {dis. }}$

The introduction of disorder is known to create new states inside the bandgap. For weak disorder, these states tend to be in close proximity to the band-edge, as shown in work involving photonic crystals ${ }^{11}$ and slow light ${ }^{16}$ waveguides. In the case of photonic crystals, it has been reported that at the band edge, the Bloch wavenumber is given by $k_{\mathrm{Bloch}}=0$; we have shown a similar behavior in the case of plasmonic waveguides ${ }^{27}$. Using the analysis in Ref. 19 , we find that the product $\Delta k_{\text {Bloch }} \times \xi \sim 0.83$, which is less than 1 and is a signature of Anderson or strong localization. A detailed explanation of this calculation is given in the Supplementary Information. The lowest-frequency resonance inside the bandgap occurs with a resonance peak at $\sim 0.62 \mathrm{THz}$ associated with a TM [201] mode ${ }^{25}$, which depends on only the aperture dimensions and not the aperture spacing, which is why the isolated modes in these two waveguides have the same resonance frequency even though they arise from waveguides with different spatial aperture patterns, albeit with identical levels of disorder. By further increasing the level of disorder $(\sigma>25 \%)$, it may be possible to realize modes deeper inside the bandgap, corresponding to larger frequencies in the input spectrum.

\section{CONCLUSION}

In summary, we have observed clear signatures of strong localization in the $\mathrm{THz}$ spectral range by using $\mathrm{THz}$ waveguides created by perforating a metal foil and introducing positional disorder into the perforations. In contrast to work performed at optical or microwave frequencies, for which low-loss materials are readily available, the relative lack of low-loss media in the far-infrared makes such observations challenging. Thus, we only observed localization with a relatively high degree of disorder. We believe that this work will encourage further investigations of localization, both theoretically and experimentally, in regimes where losses are often inherently larger than have been encountered in earlier work (for example, in the microwave and visible-frequency ranges). In such cases, the dissipation length would be an important parameter in the analysis.

\section{CONFLICT OF INTEREST}

The authors declare no conflict of interest.

\section{ACKNOWLEDGEMENTS}

This work was supported by the NSF MRSEC program at the University of Utah under grant \# DMR 1121252. SM acknowledges the Swarnajayanti Fellowship from DST, India. We thank Shayan Mookherjea and Mikhail Raikh for helpful discussions.

1 Anderson PW. Absence of diffusion in certain random lattices. Phys Rev 1958; 109: 1492-1505.

2 John S. Electromagnetic absorption in a disordered medium near a photon mobility edge. Phys Rev Lett 1984; 53: 2169-2172.

3 Pendry JB. Symmetry and transport of waves in one-dimensional disordered systems. Adv Phys 1994; 43: 461-542.

4 Segev M, Silberberg Y, Christodoulides DN. Anderson localization of light. Nat Photonics 2013; 7: 197-204.

5 Weaver RL. Anderson localization of ultrasound. Wave Motion 1990; 12: 129-142.

6 Billy J, Josse V, Zuo ZC, Bernard A, Hambrecht B et al. Direct observation of Anderson localization of matter waves in a controlled disorder. Nature 2008; 453: 891-894.

7 Garcia N, Genack AZ. Anomalous photon diffusion at the threshold of the Anderson localization transition. Phys Rev Lett 1991; 66: 1850-1853.

8 Dalichaouch R, Armstrong JP, Schultz S, Platzman PM, McCall SL. Microwave localization by two-dimensional random scattering. Nature 1991; 354: 53-55.

9 Wiersma DS, Bartolini P, Lagendijk A, Righini R. Localization of light in a disordered medium. Nature 1997; 390: 671-673.

10 Grésillon S, Aigouy L, Boccara AC, Rivoal JC, Quelin X et al. Experimental observation of localized optical excitations in random metal-dielectric films. Phys Rev Lett 1999; 82: 4520-4523.

11 Topolancik J, Ilic B, Vollmer F. Experimental observation of strong photon localization in disordered photonic crystal waveguides. Phys Rev Lett 2007; 99: 253901.

12 Schwartz T, Bartal G, Fishman S, Segev M. Transport and Anderson localization in disordered two-dimensional photonic lattices. Nature 2007; 446: 52-55.

13 Lahini Y, Avidan A, Pozzi F, Sorel M, Morandotti R et al. Anderson localization and nonlinearity in one-dimensional disordered photonic lattices. Phys Rev Lett 2008; 100: 013906.

14 Naether U, Kartashov YV, Vysloukh VA, Nolte S, Tünnermann A et al. Observation of the gradual transition from one-dimensional to two-dimensional Anderson localization. Opt Lett 2012; 37: 593-595.

15 García PD, Smolka S, Stobbe S, Lodahl P. Density of states controls Anderson localization in disordered photonic crystal waveguides. Phys Rev B 2010; 82: 165103

16 Mookherjea S, Park JS, Yang SH, Bandaru PR. Localization in silicon nanophotonic slow-light waveguides. Nat Photonics 2008; 2: 90-93.

17 Strudley T, Zehender T, Blejean C, Bakkers EPAM, Muskens OL. Mesoscopic light transport by very strong collective multiple scattering in nanowire mats. Nat Photonics 2013; 7: 413-418.

18 Bertolotti J, Gottardo S, Wiersma DS, Ghulinyan M, Pavesi L. Optical necklace states in Anderson localized 1D systems. Phys Rev Lett 2005; 94: 113903.

19 Mookherjea S, Ong JR, Luo X, Guo-Qiang L. Electronic control of optical Anderson localization modes. Nat Nanotechnol 2014; 9: 365-371.

20 Grischkowsky D, Keiding S, van Exter M, Fattinger C. Far-infrared time-domain spectroscopy with terahertz beams of dielectrics and semiconductors. J Opt Soc Am B 1990; 7: 2006-2015.

21 Jin YS, Kim GJ, Jeon SG. Terahertz dielectric properties of polymers. J Korean Phys Soc 2006; 49: 513-517.

22 Wang KL, Mittleman DM. Metal wires for terahertz wave guiding. Nature 2004; 432 : 376-379.

23 Jeon TI, Grischkowsky D. THz Zenneck surface wave (THz surface plasmon) propagation on a metal sheet. Appl Phys Lett 2006; 88: 061113.

24 Pandey S, Liu SC, Gupta B, Nahata A. Self-referenced measurements of the dielectric properties of metals using terahertz time-domain spectroscopy via the excitation of surface plasmon-polaritons. Photonics Res 2013; 1 : 148-153. 
25 Zhu WQ, Agrawal A, Nahata A. Planar plasmonic terahertz guided-wave devices. Opt Express 2008; 16: 6216-6226.

26 Fernández-Domínguez Al, Moreno E, Martin-Moreno L, García-Vidal FJ. Terahertz wedge plasmon polaritons. Opt Lett 2009; 34: 2063-2065.

27 Kumar G, Pandey S, Cui A, Nahata A. Planar plasmonic terahertz waveguides based on periodically corrugated metal films. New J Phys 2011; 13: 033024.

28 Zhu WQ, Nahata A. Electric field vector characterization of terahertz surface plasmons. Opt Express 2007; 15: 5616-5624.

29 van der Valk NCJ, Wenckebach T, Planken PCM. Full mathematical description of electro-optic detection in optically isotropic crystals. J Opt Soc Am B 2004; 21 : 622-631. (c) (i) $\odot$ This work is licensed under a Creative Commons AttributionBY NC ND NonCommercial-NoDerivs 4.0 International License. The images or other third party material in this article are included in the article's Creative Commons license, unless indicated otherwise in the credit line; if the material is not included under the Creative Commons license, users will need to obtain permission from the license holder to reproduce the material. To view a copy of this license, visit http:// creativecommons.org/licenses/by-nc-nd/4.0/

(C) The Author(s) 2017

Supplementary Information for this article can be found on the Light: Science \& Applications' website (http://www.nature.com/lsa). 